\title{
Fast Camera Imaging of Hall Thruster Ignition
}

\author{
C. L. Ellison, Y. Raitses, and N. J. Fisch
}

\begin{abstract}
Hall thrusters provide efficient space propulsion by electrostatic acceleration of ions. Rotating electron clouds in the thruster overcome the space charge limitations of other methods. Images of the thruster start-up, taken with a fast camera, reveal a bright ionization period which settles into steady-state operation over $50 \mu \mathrm{s}$. The cathode introduces azimuthal asymmetry, which persists for about $30 \mu$ s into the ignition.
\end{abstract}

Index Terms_-Plasma devices, plasma diagnostics.

$\mathbf{P}$ LASMA thrusters are used on satellites for repositioning, orbit correction, and drag compensation. The advantage of plasma thrusters over conventional chemical thrusters is that the exhaust energies are not limited by chemical energy to about an electron volt. For xenon Hall thrusters, the ion exhaust velocity can be $15-20 \mathrm{~km} / \mathrm{s}$, compared to $5 \mathrm{~km} / \mathrm{s}$ for a typical chemical thruster.

Hall thrusters overcome the space charge limit of ion thrusters. Whereas the voltage drop between the anode and cathode of an ion thruster essentially occurs in vacuum, the intervening space of a Hall thruster is filled with plasma to neutralize the otherwise limiting space charge. A radial magnetic field of approximately $200 \mathrm{G}$ prevents the axial flow of electrons, causing them to rotate azimuthally instead.

An important aspect of thruster operation on satellites, particularly because it is intermittent, is the start-up. Recently, 2-D particle-in-cell codes have modeled plasma density, potential, and current during the ignition phase [1], [2], but limited experimental evidence is available for comparison [3]. Here, we contribute time-resolved images of the thruster ignition, revealing azimuthal spatial structure and an intensity peak lasting for an ionization-relevant timescale.

Recent improvements in fast camera imaging have revealed unusual Hall thruster oscillations [4]. These were first observed in our cylindrical Hall thruster [5], but here, we study the 2-kW segmented electrode annular Hall thruster as described in [6]. Segmented electrodes are one of the ways to tailor the electric field profile in Hall thrusters [7], although here the segments remained floating. The ignition process does not use fast switching (as in [3]) but rather a steady mass flow of

Manuscript received December 1, 2010; accepted February 16, 2011. Date of publication April 5, 2011; date of current version November 9, 2011. This work was supported by the U.S. Department of Energy under Contract DEAC02-09CH11466.

The authors are with the Princeton Plasma Physics Laboratory, Princeton University, Princeton, NJ 08543 USA (e-mail: lellison@pppl.gov).

Digital Object Identifier 10.1109/TPS.2011.2121925
19 SCCM (cubic centimeter per minute at STP), an applied voltage of $250 \mathrm{~V}$ between the anode and cathode, and steady cathode heating until ignition occurs.

A Phantom v7.3 camera can acquire up to 400000 frames/s (fps). Typically, we operate at $64 \times 64$ pixel resolution and around $200 \mathrm{kfps}$, with the camera positioned about $7 \mathrm{~m}$ away and centered on the thruster axis. The large memory capacity of several seconds allows reliable capture of short-duration phenomena (such as thruster ignition), even with manual triggering. The exposure time of $4 \mu \mathrm{s}$ is sufficiently long to image the ignition of the thruster in a single capture.

Fig. 1 shows nine consecutive frames during the thruster ignition. The $64 \times 64$ image has been interpolated to larger resolution for clarity, color has been added, and the intensity has been scaled as $I^{0.75}$ to remain nearly linear while resolving a wide range of behavior within the color table. The emissions reveal a dominant intensity peak lasting about $25 \mu$ s before entering nearly steady operation. Several breathing mode frequency $(10 \mathrm{kHz})$ oscillations of above-average intensity follow the peak, but the thruster quickly settles into typical operation.

The results resemble simulations reported previously [1], [2]. PIC simulations reveal strongly peaked $(8 \times-10 \times$ steady-state mean) density and current before entering stable operation at $50 \mu \mathrm{s}$ [2]. CCD images of the plume region reveal a similar intensity peak with duration of about $25 \mu \mathrm{s}$, despite using a faster switching mechanism than our group. From the time evolution of the neutral density in [2], one may infer that the bright emissions during the early breakdown phase are from the excess neutrals present in the channel before ignition occurs.

The on-axis view shown in Fig. 1, however, reveals azimuthal structure unexplored by the 2-D PIC codes or previous imaging techniques. At $7 \mu \mathrm{s}$, the discharge can be seen to originate near the cathode, which resides at the top of the thruster. Despite the full illumination of the channel, the azimuthal disturbance persists even at $28 \mu \mathrm{s}$. The cathode introduces asymmetry by enhancing electron density and the electric field near the cathode. Considering the breakdown threshold as requiring sufficient neutral density, electron density, and electric field, it is not surprising to see the discharge originate near the cathode. Aside from the start-up, cathode effects on the electric field and thruster operation have recently been observed [8]. In our case, the rotation of the bright spot may be related to the rotating spoke observed in the cylindrical thruster [4]. Ultimately, the images suggest that understanding the start-up process requires modeling the cathode and azimuthal asymmetry.

In summary, fast camera images have been presented, measuring ignition intensity integrated axially for the first time. The images not only strongly resemble results in numerical simulation but also exhibit previously unexplored azimuthal structure. 


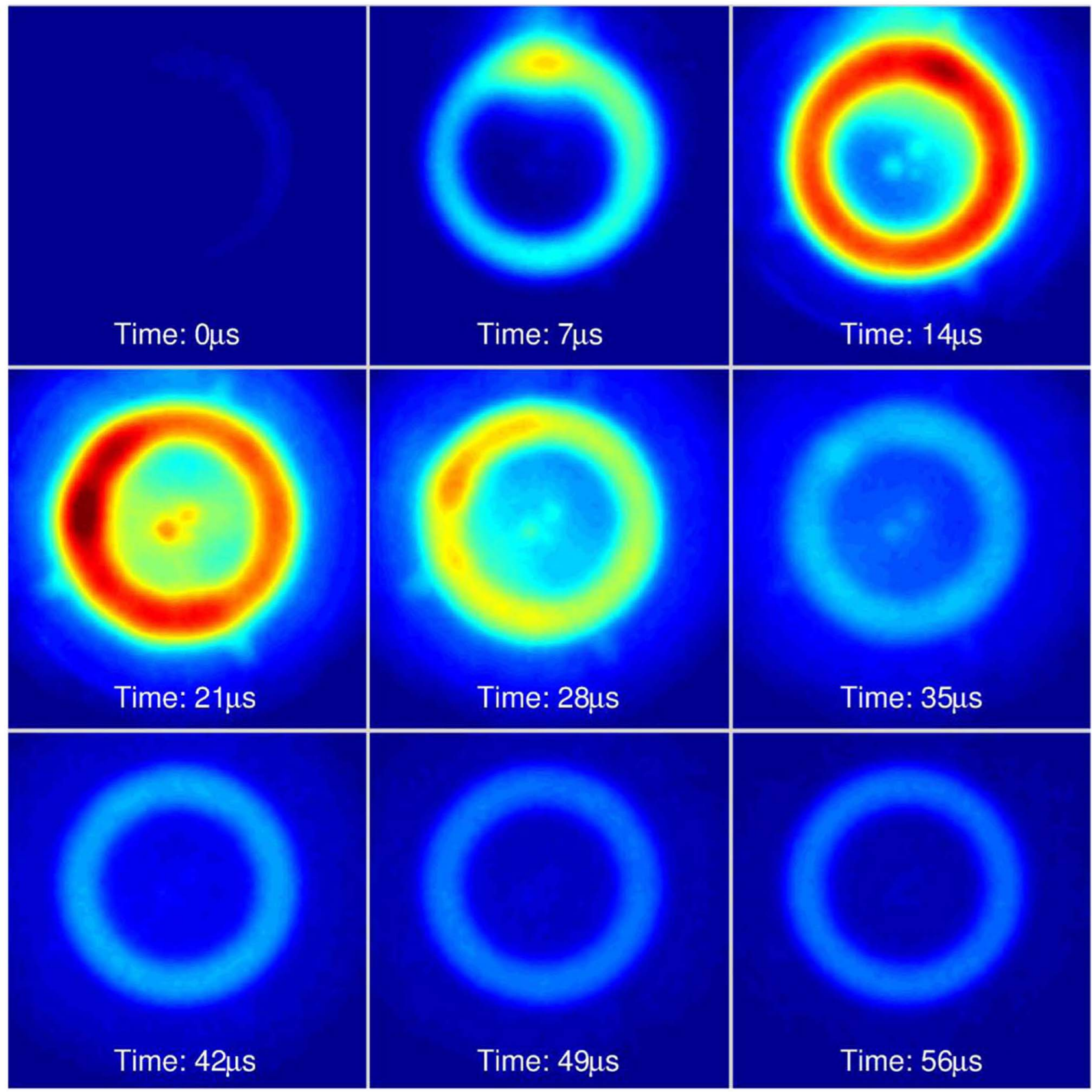

Fig. 1. Visible light emission from a xenon plasma Hall thruster. The 7- $\mu$ s snapshots with $4-\mu$ s exposure reveal the temporal behavior of Hall thruster ignition. The image is interpolated for improved resolution over the $64 \times 64$ pixels present in each frame. Color is artificial, with intensity scaled as $I^{0.75}$ to be nearly linear while enhancing the range of the color table.

\section{REFERENCES}

[1] F. Taccogna, S. Longo, M. Capitelli, and R. Schneider, "Start-up transient in a Hall thruster," Contrib. Plasma Phys., vol. 46, no. 10, pp. 781-786, Dec. 2006.

[2] H. Liu, D. R. Yu, G. J. Yan, and J. Y. Liu, "Investigation of the start transient in a Hall thruster," Contrib. Plasma Phys., vol. 48, no. 9/10, pp. 603-611, Dec. 2008.

[3] V. Vial, S. Mazouffre, M. Prioul, D. Pagnon, and A. Bouchoule, "CCD images of Hall effect thruster plume dynamics after ultrafast current ignition," IEEE Trans. Plasma Sci., vol. 33, no. 2, pp. 524-525, Apr. 2005.

[4] J. B. Parker, Y. Raitses, and N. J. Fisch, "Transition in electron transport in a cylindrical Hall thruster," Appl. Phys. Lett., vol. 97, no. 9, pp. 091501-1091501-3, Aug. 2010.
[5] Y. Raitses and N. J. Fisch, "Parametric investigation of a nonconventional Hall thruster," Phys. Plasmas, vol. 8, no. 5, pp. 2579-2586, May 2001.

[6] Y. Raitses, D. Staack, A. Duaevsky, and N. J. Fisch, "Operation of a segmented Hall thruster with low-sputtering carbon-velvet electrodes," J. Appl. Phys., vol. 99, no. 2, pp. 036103-1-036103-3, Feb. 2006.

[7] A. Fruchtman, N. J. Fisch, and Y. Raitses, "Control of the electric field profile in the Hall thruster," Phys. Plasma, vol. 8, no. 3, pp. 1048-1056, Mar. 2001

[8] G. Bourgeois, S. Mazouffre, and N. Sadeghi, "Unexpected transverse velocity component of $\mathrm{Xe}^{+}$ions near the exit plane of a Hall thruster," Phys. Plasmas, vol. 17, no. 11, pp. 113502-1-113502-6, Nov. 2010. 